\title{
Dance as treatment therapy in breast cancer patients - a systematic review
}

\section{Dança como tratamento terapêutico em pacientes com câncer de mama - uma revisão sistemática}

Leonessa Boing ; ; Aline Dandara Rafael2; Helena Braga'; Fabiana Flores Sperandio'; Alan de Jesus Pires de Moraes'; Adriana Coutinho de Azevedo Guimarães

\begin{abstract}
This systematic review aimed to identify and analyse original articles which investigated the influence of dance as adjuvant therapy in breast cancer. The selected databases were PsycInfo, PubMed, The Cochrane Library, ScienceDirect and the Virtual Health Library (VHL). The following descriptors were considered for the selection of articles: [dance therapy] OR [movement therapy] OR [complementary therapies] AND [breast cancer] OR [cancer neoplasms] OR [breast tumor] OR [breast carcinoma] present in the titles or abstracts of the articles. The quality of the data was evaluated by means of the methodological criteria proposed by Downs and Black. We identified 69 articles initially, of which only 11 were selected and analyzed. The approach followed in methodologies were dance therapy movement, Greek dance, Sacred dance, ballroom dance, classical ballet and jazz. Favorable results were found for self-image, femininity, mood, self-esteem, physical well-being, perceived stress, pain, consciousness, depression, couples trust, anxiety and fear. It was observed better range of motion and strength in upper limp, and also improvement in functional capacity. The authors pointed dance as an effective alternative adjuvant treatment in breast cancer. Dance promotes psychological benefits in women in breast cancer, as well as improvements strength and range of motion in upper limbs. Future studies are recommended with best scientific evidence, in order to investigate interventions with dance during other treatment and disease stage and with other forms of dance.
\end{abstract}

\section{Keywords}

Breast Cancer; Dancing; Therapeutics.

\section{Resumo}

Esta revisão sistemática teve como objetivo identificar e analisar os artigos originais que investigaram a influência da dança como terapia adjuvante no câncer de mama. Foram determinadas como bases de dados para as buscas: PsycInfo, PubMed, The Cochrane Library, ScienceDirect e a Biblioteca Virtual da Saúde (BVS). Os seguintes descritores foram utilizados [dance therapy] OR [movement therapy] OR [complementary therapies] AND [breast cancer] OR [cancer neoplasms] OR [breast tumor] OR [breast carcinoma] presentes nos titulos ou resumos dos trabalhos. A qualidade dos trabalhos foi investigada pela Escala metodológica Downs e Black. Foram identificados 69 artigos inicialmente, destes apenas 11 foram selecionados e analisados. As abordagens pautaram-se em metodologias da dança terapia, da dança grega, dança sagrada, dança de salão, do ballet e do jazz. Foram observados benefícios psicológicos na imagem corporal, feminilidade, humor, autoestima, bem estar físico, stress percebido, dor, consciência, depressão, confiança entre casais, ansiedade e medo. Observou-se também melhoras nos aspectos da amplitude de movimento e força dos membros superiores, bem como na capacidade funcional. Os autores apontam a dança como um tratamento adjuvante alternativo viável para mulheres com câncer de mama. A dança pode promover benefícios psicológicos em mulheres após o câncer de mama, assim como, melhora na força e amplitude de movimento dos membros superiores. Recomenda-se futuros estudos como melhor evidência cientifica, a fim de investigar a dança em outros momentos do tratamento e da doença e com outras modalidades de dança.

\section{Palavras-chave}

Câncer de mama; Dança; Terapia.

\section{Introduction}

Characterized by the World Health Organization as the most common type of cancer among women, breast cancer incidence is increasing along with the increase in life

1 Universidade do Estado de Santa Catarina. Centro de Ciências da Saúde e do Esporte. Departamento de Educação Física. Florianopolis, SC. Brasil.

2 Universidade Federal do Paraná. Programa de Residência Multiprofissional. Florianopolis, SC. Brasil. expectancy, urbanization and western lifestyle adoption ${ }^{1}$. It corresponds to $22 \%$ of new cases of cancer each year, and in 2014, there was estimated diagnosis of 57.120 new cases ${ }^{2}$. According to the same agency ${ }^{2}$ the number of deaths amounted to 13.345 (12 men and 13.225 women) for the year 2011, and in the world were computed over 508.000 women killed by the same disease ${ }^{3}$. 
The diagnosis and breast cancer antineoplastic therapy determine social, economic, physical, emotional/psychological and sexual repercussion in such patients ${ }^{4}$. In relation to physical changes, women have varying degrees of disabilities ${ }^{5}$ such as discomfort, pain, reduced functional capacity, lymphedema and fatigue $e^{6-8}$. Regarding to emotional aspects, treatment can implies in feelings as angry, sadness, restlessness, anxiety, anguish, fear, and grief ${ }^{10}$ which can affects the body image, self-esteem and functional loss 9 .

There are enough enhancements which corroborate the positive effects arising from the practice of physical exercises in individuals with breast cancer ${ }^{11}$. Overall scientific evidence suggests that exercise is safe and feasible to be applied as non-pharmacological intervention for the treatment of cancer and it can also improve aspects such as quality of life and psychophysiological function ${ }^{12,13}$. Consistent results include how exercise effects the reduction in fatigue condition ${ }^{14}$, postural changes, on muscular fibrosis and/or tissue adhesion of surgical area ${ }^{15}$, in depression, anxiety and anguish, combined with positive changes in parameters of physical fitness, aerobic capacity and general muscle function ${ }^{13,16}$.

The dance application is pointed out in several studies as one of the possibilities of adjuvant therapies, as in cancer patients ${ }^{14}$ hemophilia patients ${ }^{17}$, elderly with depression ${ }^{18}$ and people with Parkinson disease ${ }^{19}$. Dance can be beneficial for enhancing emotional and social aspects in the cancer patients life $^{14}$, and also alternative sports therapy programme for patients with haemophilia ${ }^{17}$, after showing positive results in pain. When regarding to pshychological aspects, dance can be associated with decrease depression symptons in elderly patients and also fewer prescriptions of antidepressants ${ }^{18}$. Dance can be a possibility of exercise intervention in different patients ${ }^{14,17,18,19}$. Considering the effects of the breast cancer treatment and all the collateral effects, dance as therapeutic context can be beneficial for this population. Given such context, the effects of dance as an adjuvant therapy in breast cancer patients treatment are not yet clear, and as such, identification and analyzis is proposed for the influence of dance as adjuvant therapy in breast cancer.

\section{Methods}

\section{Type of study}

The present study is characterized as a systematic review, as it intends to gather all the evidence that fits the specified eligibility criteria in order to answer a specific research question ${ }^{20}$.

\section{Search strategy}

This systematic review was registered at PROSPERO (International Prospective Register of Systematic Reviews) under the number CRD42016047316 and the development of the review was carried out following the PRISMA model - Preferred Reporting Items for Systematic Reviews and Meta-Analysis ${ }^{21}$. The selected databases were: PsycInfo, PubMed, The Cochrane Library, ScienceDirect and the Virtual Health Library (VHL). The following descriptors were considered for the selection of articles: [dance] OR [movement therapy] OR [complementary therapies] AND [breast cancer] OR [cancer neoplasms] OR [breast tumor] OR [breast carcinoma] present in the titles or abstracts of the articles. Also, a manual search was conducted in the reference list of the articles. The systematic search was carried out from February to March 2017.

The guiding question that led the selection of articles, as well as their later analysis was according to the PICOS referencial: How are structured the dance intervention in breast cancer women and what are the possible benefits of the intervention? 


\section{Criteria for selection of articles}

As selection criteria, articles that approached dance as adjuvant therapy in breast cancer female patients with more than 18 years old were included. The inclusion criteria were defined as: (a) original articles as clinical trial, quasi-esperimental or case studies published in English, Portuguese or Spanish; (b) all involving the descriptors listed in as its variables; (c) published in indexed journals with Qualis from Coordination of Improvement of Higher Level Personnel - CAPES and/or impact factor; (d) available in digital format. Non inclusion criteria were considered as follows: (a) systematic reviews, dissertations or thesis; (b) duplicate references.

\section{Study analysis}

In the first phase of the study titles and abstracts were identified. After deleted articles that did not meet the criteria for inclusion, along with the duplicate articles, a file process was carried out of all selected articles, for full reading. All titles and abstracts retrieved by the electronic search were analysed manually by two researchers in a blind and independent manner considering that the differences found were settled through consensus between the researchers. After completion of the selection, the full reading of the documents was carried out, and the data extraction were developed by two researchers. In each article were observed the study design, objective, sample, location, age, duration of the intervention, elements of the intervention (protocol of the intervention), evaluation tools and main results.

\section{Risk of bias evaluation}

The quality of the data was evaluated by means of the methodological criteria proposed by Downs e Black ${ }^{22}$, scored on 27 issues of a checklist, focusing on methodological aspects of communication, external validity, internal validity (bias), internal validity (confounding variables), and statistical power. For each question, the score zero was applied, in case the article was not concerning the evaluation subject, and the score one, when a positive reply was observed to the demand, considering that only question five had the maximum score two. The maximum score for each article was 28 points.

\section{Results}

Figure 1 presents the study selection flowchart. After the initial search, 69 studies were found using the descriptors defined in the methodology, considering 16 in details in Virtual Health Library (VHL), 10 in PscyInfo, 27 in PubMed, eight in The Cochrane Library, seven in Science Direct and one in Scielo. The following articles were selected: 63 in English, two in Spanish, three in German and one in Portuguese. At first, 23 studies were excluded because they consisted of duplicate references. Then, 20 studies were excluded: 15 for being systematic reviews, one for being characterized as editorial, two for being thesis, and two for not allowing access. After reading the full article 15 were excluded for being off the theme established in the delimitations of the study, which left 11 articles included faithfully in this systematic review.

As regards the methodological review of the articles, by means of the scale Downs e Black ${ }^{22}$, the average score was 18 points, with a minimum of eight and maximum of 23 points. The eight point score refers to the study of Molinaro et al. ${ }^{23}$, as a clinical case report does not present statistical purposes, making it impossible to make a evaluation of results, which also happens to studies that use qualitative analyses, such as Serlin et al. ${ }^{32}$, and Blázquez et al. ${ }^{27}$. Blinding was one of the characteristics that had lower scores in the evaluation. Another factor con- 
sidered low score is the generalization of the results. If studies of poor methodological quality were excluded, the results would continue to indicate dancing as a beneficial physical activity in women with breast cancer. However, further studies with randomized methodology and blinding of participants and researchers should be encouraged.

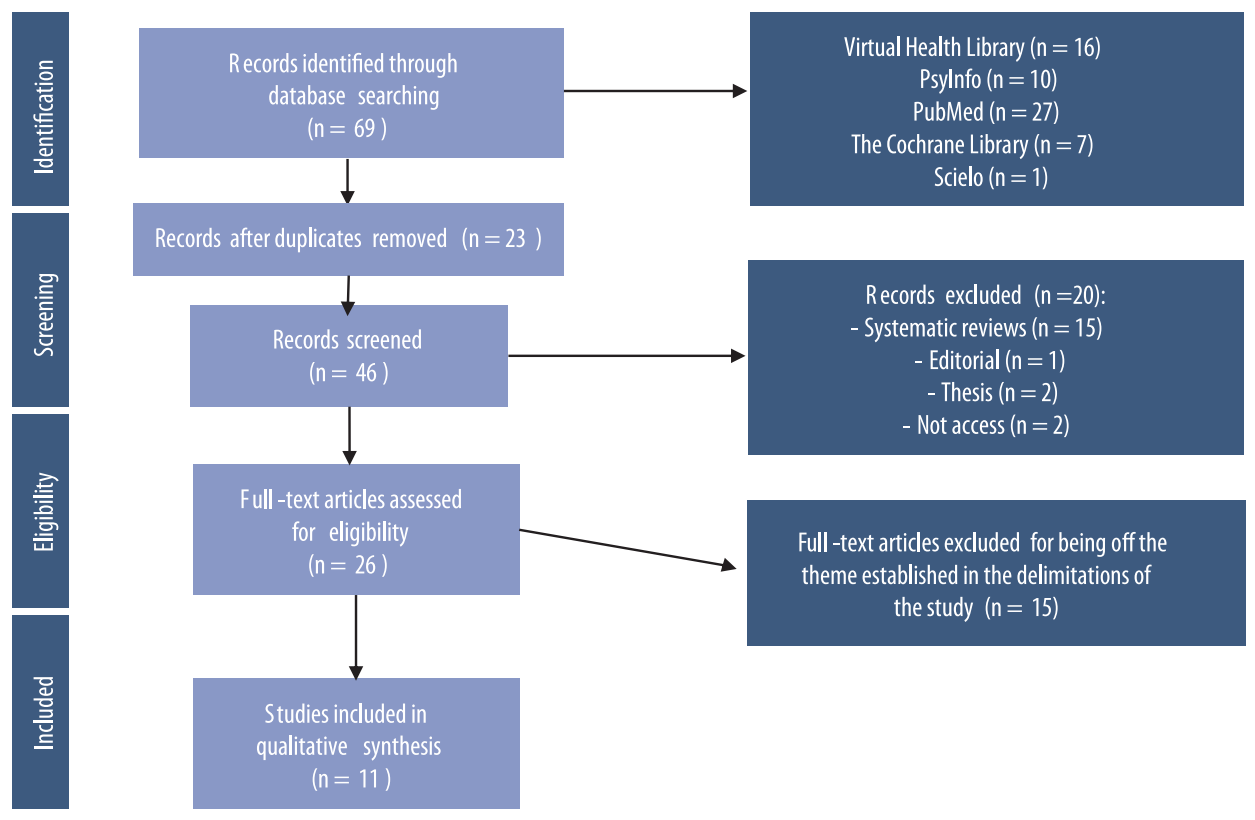

FIGURE 1 - PRISMA21 Study selection flow diagram.

\section{Descriptive synthesis}

In table 1, detailed information of the 11 articles, such as study design, objective, sample, location, age, duration of the intervention, elements of the intervention (protocol of the intervention), evaluation tools and the scale score Downs and Black $(1998)^{22}$. In relation to the design of the study, all reported such criterion, namely: randomized clinical trial $(\mathrm{n}=7)$, followed by quasi-experimental $(\mathrm{n}=3)$ and case study $(\mathrm{n}=1)$. The sample ranged from one to 139 patients in different age groups, totaling 482 women involved.

As for the dance modality used, the divergence was found in the studies. Reports of interventions were found within the modalities of jazz, classical ballet ${ }^{23}$ and Greek dance ${ }^{24}$, sacred dance ${ }^{25}$ and ballrrom dance ${ }^{26}$. Nevertheless, the other seven articles described their intervention protocols based on techniques of dance/movement therapy ${ }^{27-33}$. There was a variation in terms of the intervention period (three weeks to 24 weeks), weekly rate (one to three sessions a week) and duration of the session (one to three hours).

The results of the articles were described in Table 2. Research variables were categorized into psychological and physical in all included studies ${ }^{23-33}$, but investigation on both physical and physiological variables were identified only in four studies ${ }^{23-26}$.

In relation to psychological aspects only, all 11 studies investigated these variables. Favorable results were found for social functioning $(\mathrm{p}=0.040)$, mental health $(p=0.040)$ and role emotional $(p=0.070)^{26}$, welfare aspect and $\operatorname{mood}(p<0.050)^{29}$ quality of life on psychological $(p=0.001)$ and environment $(p=0.029)^{25}$, general quality of life $(p<0.050)$, body image $(p<0.010)^{31}$, improvements in satisfaction with life $(p=0.001)$ and also a reduction in depressive symptoms $(p=0.010)^{24}$, fear of recurrence $(p=0.020)^{28}$, perceived stress $(p<0.050)$, pain severity and pain 
interference $(p<0.050)^{30}$, mental component of quality of life $(p=0.001)$, vitality $(\mathrm{p}=0.004)$, social functioning $(\mathrm{p}=0.040)$, and mental health $(\mathrm{p}=0.040)^{26}$.

Some studies presented only qualitative perceptions on psychological variables, and statistical calculations were not carried. Two of them present benefits in relation to body image $e^{23,33}$, and other two promoted improvement in mental health as alleviation of depression and anxiety ${ }^{27,32}$.

Eight studies investigated physical variables and showed important results after intervention with dance. Were found improvements in functional capacity ( $\mathrm{p}$ $=0.030)$, physical activity $(\mathrm{p}=0.010)^{26}$, force and fatigue $(\mathrm{p}<0.050)^{29}$, range of motion of the arm affected $(p<0.001)^{31}$, physical function $(p=0.001)$, left and right hand grip strength $(\mathrm{p}=0.001)$, volume of the left $\operatorname{arm}(\mathrm{p}=0.028)^{24}$, and physical aspects of quality of life $(\mathrm{p}<0.001)^{25}$. Statistical calculations were not carried out in some studies, but they presented some qualitative improvemets in physical variables as Serlin et al..$^{32}$ in fatigue, vigor and tension, Blázquez et al. ${ }^{27}$ in physical aspects, Ho et al. ${ }^{33}$ improvement in total functioning and physical symptoms.

TABLE 1 - Detailed analysis of selected articles about dance in breast cancer women in relation to the type of study, objective, sample, intervention length, intervention elements and evaluation tools $(n=11)$.

\begin{tabular}{|c|c|c|c|c|c|c|c|}
\hline Reference & Study design & Objective & $\begin{array}{l}\text { Sample/Age/ } \\
\text { Location }\end{array}$ & $\begin{array}{l}\text { Intervention } \\
\text { length }\end{array}$ & $\begin{array}{l}\text { Intervention } \\
\text { elements (Protocol } \\
\text { of intervention) }\end{array}$ & Evaluation tools & $\begin{array}{l}{ }^{*} \text { Downs } \\
\text { and Black }\end{array}$ \\
\hline $\begin{array}{l}\text { Molinaro } \\
\text { et al. }{ }^{23}\end{array}$ & $\begin{array}{l}\text { Clinical Report } \\
\text { - Quasi- } \\
\text { experimental. }\end{array}$ & $\begin{array}{l}\text { Describe a dance } \\
\text { exercise program for } \\
\text { breast cancer women } \\
\text { who had undergone } \\
\text { mastectomy or } \\
\text { Lumpectomy. }\end{array}$ & $\begin{array}{l}37 \text { women who } \\
\text { underwent } \\
\text { mastectomy in } \\
\text { treatment or post } \\
\text { treatment } \\
\text { (30 to } 81 \text { years). } \\
\text { Philadelphia } \\
\text {-United States. }\end{array}$ & $\begin{array}{l}\text { Two sessions } \\
\text { a week, one } \\
\text { hour class. }\end{array}$ & $\begin{array}{l}\text { Dance program } \\
\text { which combined } \\
\text { classical ballet and } \\
\text { jazz with functional } \\
\text { exercises. }\end{array}$ & $\begin{array}{l}\text { Heart rate, range } \\
\text { of motion, active } \\
\text { muscle strength, } \\
\text { pain, skin changes, } \\
\text { circumference of } \\
\text { upper limb, posture } \\
\text { and balance. }\end{array}$ & 8 \\
\hline $\begin{array}{l}\text { Dibbell- } \\
\text { Hope }^{29}\end{array}$ & $\begin{array}{l}\text { Randomized } \\
\text { clinical study study } \\
\text { with a quantitative } \\
\text { and qualitative } \\
\text { phenomenological } \\
\text { approach. }\end{array}$ & $\begin{array}{l}\text { The study is intended } \\
\text { to answer the } \\
\text { following question: } \\
\text { Can the Authentic } \\
\text { Movement technique } \\
\text { help breast cancer } \\
\text { women to improve } \\
\text { their psychological } \\
\text { domains to adapt } \\
\text { to the illness and its } \\
\text { treatment, and can } \\
\text { this improvement be } \\
\text { maintained over time? }\end{array}$ & $\begin{array}{l}33 \text { women with } \\
\text { breast cancer } \\
\text { stage I or II in post } \\
\text { treatment of } 6 \text { or } \\
60 \text { months divided } \\
\text { into experimental } \\
\text { and control group. } \\
\text { (35 to } 80 \text { years) } \\
\text { San Francisco } \\
\text { Bay Area -United } \\
\text { States. }\end{array}$ & $\begin{array}{l}\text { Six weeks, } \\
\text { one session a } \\
\text { week, three } \\
\text { hours. }\end{array}$ & $\begin{array}{l}\text { Authentic } \\
\text { Movement } \\
\text { Group - dance } \\
\text { practice in which } \\
\text { the practitioners } \\
\text { move on their own } \\
\text { time, at their pace, } \\
\text { on their impulse } \\
\text { and as enjoyable } \\
\text { as possible for the } \\
\text { body. }\end{array}$ & $\begin{array}{l}\text { Questions about } \\
\text { personal experience } \\
\text { in relation to } \\
\text { diagnosis (qualitative). } \\
\text { Questionnaires: Mood } \\
\text { (Profile of Mood States), } \\
\text { distress (Symptom } \\
\text { Checklist 90-Revised), } \\
\text { body image and self- } \\
\text { esteem (Borscheid, } \\
\text { Walster, Bohrnstedt } \\
\text { Body Image Scale- } \\
\text { BWB), all applied pre } \\
\text { and post intervention } \\
\text { (quantitative). }\end{array}$ & 19 \\
\hline $\begin{array}{l}\text { Serlin et } \\
\text { al. }^{32}\end{array}$ & $\begin{array}{l}\text { Quasi-experimental } \\
\text { study. Quantitative } \\
\text { and qualitative } \\
\text { phenomenological } \\
\text { approach. }\end{array}$ & $\begin{array}{l}\text { Investigate the } \\
\text { effectiveness of dance/ } \\
\text { movement therapy } \\
\text { in psychosocial and } \\
\text { spiritual adaptation in } \\
\text { breast cancer women. }\end{array}$ & $\begin{array}{l}95 \text { women post- } \\
\text { breast cancer. } \\
\text { Above } 18 \text { years old. } \\
\text { California - United } \\
\text { States. }\end{array}$ & $\begin{array}{l}12 \text { weeks, one } \\
\text { session per } \\
\text { week during } \\
45 \text { minutes. }\end{array}$ & $\begin{array}{l}\text { Kinaesthetic } \\
\text { imagining form } \\
\text { of dance therapy. } \\
\text { Involving imagery, } \\
\text { meditation and } \\
\text { movement. }\end{array}$ & $\begin{array}{l}\text { Qualitative interviews } \\
\text { about dance } \\
\text { effectiveness in } \\
\text { psychosocial and } \\
\text { spiritual adaptation. }\end{array}$ & \# \\
\hline $\begin{array}{l}\text { Sandel et } \\
\text { al. }^{31}\end{array}$ & $\begin{array}{l}\text { Randomized } \\
\text { Clinical Study. Pilot } \\
\text { Study. }\end{array}$ & $\begin{array}{l}\text { Determine the effect } \\
\text { of movement and } \\
\text { dance program on } \\
\text { quality of life and } \\
\text { shoulder functionality } \\
\text { in breast cancer } \\
\text { women and reated } \\
\text { within the previous } \\
\text { five years. }\end{array}$ & $\begin{array}{l}35 \text { women with } \\
\text { breast cancer } \\
\text { divided into } \\
\text { intervention group } \\
(n=19) \text { and } \\
\text { control group ( } n= \\
\text { 16) }(61.0 \text { years old). } \\
\text { Connecticut - } \\
\text { United States }\end{array}$ & $\begin{array}{l}12 \text { weeks: } \\
\text { two sessions } \\
\text { per week in } \\
\text { the first six } \\
\text { weeks and } \\
\text { one session } \\
\text { per week for } \\
\text { the other } \\
\text { six weeks, } \\
\text { totaling } 18 \\
\text { sessions. }\end{array}$ & $\begin{array}{l}\text { The interventions } \\
\text { were divided into } \\
\text { warming up ( } 10 \\
\text { to } 15 \text { minutes), } \\
\text { basic exercises (for } \\
\text { premium members), } \\
\text { dance moves ( } 25 \\
\text { to } 30 \text { minutes), } \\
\text { relaxation/back to } \\
\text { calm (10 minutes). } \\
\text { The Lebed Method } \\
\text { was used here. }\end{array}$ & $\begin{array}{l}\text { Quality of life (SF-36 } \\
\text { and FACT-B), Body } \\
\text { Image Scale, range } \\
\text { of motion (ROM) of } \\
\text { shoulder and upper } \\
\text { limb circumference. }\end{array}$ & 23 \\
\hline
\end{tabular}


... continue

\begin{tabular}{|c|c|c|c|c|c|c|c|}
\hline Reference & Study design & Objective & $\begin{array}{l}\text { Sample/Age/ } \\
\text { Location }\end{array}$ & $\begin{array}{l}\text { Intervention } \\
\text { length }\end{array}$ & $\begin{array}{l}\text { Intervention } \\
\text { elements (Protocol } \\
\text { of intervention) }\end{array}$ & Evaluation tools & $\begin{array}{l}\text { *Downs } \\
\text { and Black }\end{array}$ \\
\hline $\begin{array}{l}\text { Blasquez } \\
\text { et al. }{ }^{27}\end{array}$ & $\begin{array}{l}\text { Case Study with } \\
\text { a qualitative } \\
\text { approach. }\end{array}$ & $\begin{array}{l}\text { Describe the } \\
\text { therapeutic process } \\
\text { and the possible } \\
\text { influences of mirror } \\
\text { neuron in kinesthetic } \\
\text { empathy through } \\
\text { the use of movement } \\
\text { and dance therapy } \\
\text { for breast cancer. }\end{array}$ & $\begin{array}{l}\text { A 32-year-old } \\
\text { patient diagnosed } \\
\text { with breast cancer. } \\
\text { Barcelona-Spain. }\end{array}$ & $\begin{array}{l}\text { Five months, } \\
20 \text { sessions, } \\
\text { one hour } \\
\text { weekly. }\end{array}$ & $\begin{array}{l}\text { The participant } \\
\text { was subjected to } \\
\text { a open program } \\
\text { of dance and } \\
\text { movement therapy. }\end{array}$ & $\begin{array}{l}\text { Qualitative clinical } \\
\text { notes from patients } \\
\text { and therapist. }\end{array}$ & \# \\
\hline $\begin{array}{l}\text { Kaltsatou } \\
\text { et al. } .^{24}\end{array}$ & $\begin{array}{l}\text { Randomized } \\
\text { clinical trial. }\end{array}$ & $\begin{array}{l}\text { Evaluate the } \\
\text { influence of a mixed } \\
\text { exercise program, } \\
\text { including Greek } \\
\text { traditional dances } \\
\text { and upper body } \\
\text { training, in physical } \\
\text { function, strength } \\
\text { and psychological } \\
\text { condition of breast } \\
\text { cancer survivors. }\end{array}$ & $\begin{array}{l}27 \text { post-breast } \\
\text { cancer women; } \\
\text { the experimental } \\
\text { group }(n=14) \\
\text { and control group } \\
(n=13) .(56.6 \\
\text { years old) } \\
\text { Thessaloniki - } \\
\text { Greece. }\end{array}$ & $\begin{array}{l}24 \text { weeks, } \\
\text { three week } \\
\text { sessions, one } \\
\text { hour each. }\end{array}$ & $\begin{array}{l}60 \text { min session } \\
\text { with: warm-up, } \\
\text { aerobic training } \\
\text { with Greek dance, } \\
\text { training of upper } \\
\text { limbs and returns } \\
\text { to calm. }\end{array}$ & $\begin{array}{l}\text { Blood pressure, } \\
\text { heart rate, 6-minute } \\
\text { walk test, hand grip } \\
\text { (dynamometer), arm } \\
\text { volume, Depression } \\
\text { (BECK Inventory) } \\
\text { and Life Satisfaction } \\
\text { Inventory (LSF). }\end{array}$ & 21 \\
\hline $\begin{array}{l}\text { Crane- } \\
\text { Okada et } \\
\text { al. }{ }^{28}\end{array}$ & $\begin{array}{l}\text { Randomized } \\
\text { controlled pilot } \\
\text { feasibility study. }\end{array}$ & $\begin{array}{l}\text { Test feasibility and } \\
\text { shortterm effects of } \\
\text { a 12-week Mindful } \\
\text { Movement Program } \\
\text { (MMP) intervention } \\
\text { combining } \\
\text { mindfulness with } \\
\text { self-directed } \\
\text { movement on } \\
\text { quality of life and } \\
\text { mindfulness in } \\
\text { older female breast } \\
\text { cancer survivors after } \\
\text { treatment. }\end{array}$ & $\begin{array}{l}49 \text { post-breast } \\
\text { cancer women, } \\
\text { divided in } \\
\text { experimental } \\
\text { group }(n=30) \\
\text { and control group } \\
(\mathrm{n}=19) ;(50 \text { to } 90 \\
\text { years). } \\
\text { Los Angeles } \\
\text {-United States }\end{array}$ & $\begin{array}{l}12 \text { weeks, } \\
\text { one session } \\
\text { per week, } \\
\text { two hours } \\
\text { each session. }\end{array}$ & $\begin{array}{l}\text { Session of Mindful } \\
\text { Movement } \\
\text { Program (MMP) } \\
\text { walks, talks, } \\
\text { exploring specific } \\
\text { bodyparts, small } \\
\text { movements, } \\
\text { energetic and } \\
\text { active movements, } \\
\text { pairwork. }\end{array}$ & $\begin{array}{l}\text { Quality of life (Quality } \\
\text { of Life Breast Cancer); } \\
\text { Physical (Symptom } \\
\text { Bother Scale); Body } \\
\text { image (Body Image), } \\
\text { Psychological aspects } \\
\text { (Hospital Anxiety and } \\
\text { Depression } \\
\text { Scale; Fear of } \\
\text { Recurrence Scale); } \\
\text { Individual diary, } \\
\text { mental alertness } \\
\text { (Mindful Attention } \\
\text { awareness Scale), } \\
\text { emotional (Self } \\
\text { Compassion Scale), } \\
\text { cognitive (Five Facet } \\
\text { Mindfulness } \\
\text { Questionnaire), } \\
\text { attitude (Experiences } \\
\text { Questionnaire). }\end{array}$ & 19 \\
\hline $\begin{array}{l}\text { Frison et } \\
\text { al. } .^{25}\end{array}$ & $\begin{array}{l}\text { Quasi- } \\
\text { experimental pilot } \\
\text { study. }\end{array}$ & $\begin{array}{l}\text { Evaluate the life } \\
\text { quality of women } \\
\text { who underwent } \\
\text { mastectomy that } \\
\text { participated in the } \\
\text { study (S) and in } \\
\text { the control group } \\
\text { (C) carried out } \\
\text { before and after the } \\
\text { application of sacred } \\
\text { dance. }\end{array}$ & $\begin{array}{l}35 \text { mastecomized } \\
\text { women, study } \\
\text { group }(n=19) \\
\text { and control group } \\
(n=24)(52,4 \\
\text { years old). } \\
\text { Campinas - Brazil. }\end{array}$ & $\begin{array}{l}\text { Three } \\
\text { months, } \\
\text { once a week, } \\
\text { one hour } \\
\text { session. }\end{array}$ & $\begin{array}{l}\text { The participants } \\
\text { received sacred } \\
\text { dance classes. }\end{array}$ & $\begin{array}{l}\text { Quality of Life (World } \\
\text { Health Organization } \\
\text { Quality of Life, } \\
\text { WHOQOL - bref) } \\
\text { and a Demographic } \\
\text { Questionnaire. }\end{array}$ & 19 \\
\hline Ho et al..$^{30}$ & $\begin{array}{l}\text { Randomized } \\
\text { controlled single } \\
\text { blind trial. }\end{array}$ & $\begin{array}{l}\text { Investigate the } \\
\text { effectiveness of } \\
\text { dance movement } \\
\text { therapy (DMT) on } \\
\text { improving treatment- } \\
\text { related symptoms } \\
\text { in a randomized } \\
\text { controlled trial. }\end{array}$ & $\begin{array}{l}139 \text { chinese } \\
\text { patients } \\
\text { with breast } \\
\text { cancer during } \\
\text { radiotherapy. } \\
\text { Divided in DMT } \\
\text { group }(n=69) \text {, } \\
\text { and waitlist } \\
\text { control group ( } n \\
=70) \text {. (50.0 years } \\
\text { old) } \\
\text { Hong Kong - } \\
\text { China. }\end{array}$ & $\begin{array}{l}\text { Three weeks, } \\
\text { twice a } \\
\text { week, } 90 \\
\text { minutes a } \\
\text { session. }\end{array}$ & $\begin{array}{l}\text { The program } \\
\text { consists of } \\
\text { stretching, } \\
\text { relaxation exercises, } \\
\text { movement games } \\
\text { and rhythmic } \\
\text { body movement } \\
\text { to exercise the } \\
\text { upper extremities, } \\
\text { improvisational } \\
\text { dance and } \\
\text { movement to } \\
\text { explore positive } \\
\text { emotions. }\end{array}$ & $\begin{array}{l}\text { Stress (Perceived } \\
\text { Stress Scale); Pain (The } \\
\text { Brief Pain Inventory); } \\
\text { Sleep Disturbance } \\
\text { (The Pittsburgh Sleep } \\
\text { Quality Index); Quality } \\
\text { of Life (The Functional } \\
\text { Assessment of Cancer } \\
\text { Therapy - Breast). }\end{array}$ & 21 \\
\hline
\end{tabular}




\begin{tabular}{|c|c|c|c|c|c|c|c|}
\hline Reference & Study design & Objective & $\begin{array}{l}\text { Sample/Age/ } \\
\text { Location }\end{array}$ & $\begin{array}{l}\text { Intervention } \\
\text { length }\end{array}$ & $\begin{array}{l}\text { Intervention } \\
\text { elements (Protocol } \\
\text { of intervention) }\end{array}$ & Evaluation tools & $\begin{array}{l}\text { *Downs } \\
\text { and Black }\end{array}$ \\
\hline Ho et al. ${ }^{33}$ & $\begin{array}{l}\text { Randomized } \\
\text { controlled single } \\
\text { blind trial with } \\
\text { qualitative } \\
\text { approach. }\end{array}$ & $\begin{array}{l}\text { Qualitatively explore } \\
\text { the beneficial } \\
\text { elements of DMT and } \\
\text { how the intervention } \\
\text { was able or not } \\
\text { able to meet the } \\
\text { needs of patients } \\
\text { over the course of } \\
\text { radiotherapy. }\end{array}$ & $\begin{array}{l}104 \text { breast cancer } \\
\text { patients divided } \\
\text { in Concurrent } \\
\text { Radiotherapy (RT) } \\
\text { ( } \mathrm{n}=60 \text { ) and Post } \\
\text { Radiotherapy ( } \mathrm{n} \\
=44) \text {. ( } 50.0 \text { years } \\
\text { old) } \\
\text { Hong Kong - } \\
\text { China. }\end{array}$ & $\begin{array}{l}\text { Three weeks, } \\
\text { twice a } \\
\text { week, } 90 \\
\text { minutes a } \\
\text { session. }\end{array}$ & $\begin{array}{l}\text { The program } \\
\text { consists of stretching, } \\
\text { relaxation exercises, } \\
\text { movement games } \\
\text { and rhythmic } \\
\text { body movement } \\
\text { to exercise the } \\
\text { upper extremities, } \\
\text { improvisational } \\
\text { dance and } \\
\text { movement to explore } \\
\text { positive emotions. }\end{array}$ & $\begin{array}{l}\text { Two qualitative } \\
\text { questions: "Did } \\
\text { you find the DMT class } \\
\text { helpful to you or not? } \\
\text { If so, in what aspects?" } \\
\text { and "Was the DMT } \\
\text { class helpful during } \\
\text { your radiotherapy } \\
\text { treatment? If so, in } \\
\text { what aspects?" }\end{array}$ & 21 \\
\hline $\begin{array}{l}\text { Pisu et } \\
\text { al. }^{26}\end{array}$ & $\begin{array}{l}\text { Pilot randomized } \\
\text { controlled trial } \\
\text { with two arms. }\end{array}$ & $\begin{array}{l}\text { Assess the feasibility, } \\
\text { acceptability, and } \\
\text { impact of a ballroom } \\
\text { dance intervention } \\
\text { on improving quality } \\
\text { of life (QOL) and } \\
\text { relationship outcomes } \\
\text { in cancer survivors } \\
\text { and their partners. }\end{array}$ & $\begin{array}{l}31 \text { women cancer } \\
\text { survivors (68\% } \\
\text { breast cancer) } \\
\text { and their partners. } \\
\text { (57.9 years old). } \\
\text { Birmingham - } \\
\text { United States. }\end{array}$ & $\begin{array}{l}12 \text { weeks } \\
\text { including } \\
10 \text { private } \\
\text { weekly } \\
\text { dance } \\
\text { lessons and } \\
\text { two practice } \\
\text { parties. }\end{array}$ & $\begin{array}{l}\text { Light intensity } \\
\text { ballroom dance in } \\
\text { couples. Including: } \\
\text { Foxtrot; Waltz; Cha- } \\
\text { Cha and East coast } \\
\text { swing. }\end{array}$ & $\begin{array}{l}\text { Physical activity (Godin } \\
\text { Leisure-Time } \\
\text { Exercise Questionnaire); } \\
\text { Functional capacity } \\
\text { (6 Minute Walk Test); } \\
\text { Quality of Life (SF-36); } \\
\text { Couples' trust (Dyadic } \\
\text { Trust Scale), and other } \\
\text { dyadic outcomes. }\end{array}$ & 19 \\
\hline
\end{tabular}

* Points from the methodologic evaluation of the studies by Downs and Black Scale.

TABLE 2 - Detailed analysis of the selected articles in relation to main results in psychological and physical variables after a dance intervention in breast cancer women.

\begin{tabular}{|c|c|c|}
\hline \multirow{2}{*}{ Reference } & \multicolumn{2}{|l|}{ Main results } \\
\hline & Psychological variables & Physical variables \\
\hline $\begin{array}{l}\text { Molinaro et } \\
\text { al. } .^{23}\end{array}$ & $\begin{array}{l}\text { A dance program for these patients is a way of encouraging the practice of } \\
\text { continued exercise, in addition to promoting feelings of femininity, grace } \\
\text { and sexuality in relation to body image, which may be threatened by the } \\
\text { nature of the surgery. In addition, the group provides a support system for } \\
\text { these patients. Statistical calculations were not carried out. }\end{array}$ & Not investigated. \\
\hline $\begin{array}{l}\text { Dibbell- } \\
\text { Hope }^{29}\end{array}$ & $\begin{array}{l}\text { The experimental group presented improvement in somatization variables } \\
\text { within the welfare aspect }(\mathrm{p}<0.050) \text {. In the quantitative results changes } \\
\text { in mood and in distress were noticed. The qualitative results were positive, } \\
\text { compared with mood, anxiety, body image, self-esteem and social support. }\end{array}$ & $\begin{array}{l}\text { The experimental group presented improvement in force } \\
\text { and fatigue aspect }(p<0.050) \text {. }\end{array}$ \\
\hline Serlin et al. ${ }^{32}$ & $\begin{array}{l}\text { Depression and anxiety were alleviated. Statistical calculations were not } \\
\text { carried out. }\end{array}$ & $\begin{array}{l}\text { Significant improvements were found in fatigue, vigor } \\
\text { and tension. Statistical calculations were not carried out. }\end{array}$ \\
\hline $\begin{array}{l}\text { Sandel et } \\
\text { al. }{ }^{31}\end{array}$ & $\begin{array}{l}\text { The dance movement program substantially improved the quality of life } \\
\text { in patients with breast cancer }(p<0.050) \text { however; the body images ( } p \\
<0.001) \text { also received improvements, but have not been proven to be } \\
\text { resulting from the intervention. }\end{array}$ & $\begin{array}{l}\text { The range of motion of the arm affected }(p<0.001) \\
\text { received improvements, but have not been proven to be } \\
\text { resulting from the intervention. }\end{array}$ \\
\hline $\begin{array}{l}\text { Blasquez et } \\
\text { al. } .^{27}\end{array}$ & $\begin{array}{l}\text { The participant qualitatively reported improvement in her mental health } \\
\text { perception. Statistical calculations were not carried out. }\end{array}$ & $\begin{array}{l}\text { The participant presented improvement in her physical } \\
\text { qualitatively reported welfare. Statistical calculations were } \\
\text { not carried out. }\end{array}$ \\
\hline $\begin{array}{l}\text { Kaltsatou et } \\
\text { al. } .^{24}\end{array}$ & $\begin{array}{l}\text { The experimental group revealed improvements in satisfaction with life ( } p= \\
0.001) \text { and also a reduction in depressive symptoms }(p=0.001) \text {. }\end{array}$ & $\begin{array}{l}\text { The results showed significant increase in physical } \\
\text { function }(p=0.001) \text { and left and right hand grip strength } \\
\text { ( } p=0.001) \text { in the experimental group, after the training } \\
\text { program. Still, significant reductions were found in the } \\
\text { volume of the left arm }(p=0.028) \text {. }\end{array}$ \\
\hline
\end{tabular}

Crane- $\quad$ After the intervention, the participants in the Experimental Group (EG)

Crane- 28 showed improvement in quality of life through a reduction in the fear of recurrence $(p=0.020)$ and improvement in mindfulness attitude $(p=0.026)$.

Frison et al. ${ }^{25}$ After the intervention the study group $(S)$ demonstrate improvement in psychological $(p=0.001)$ and environment $(p=0.029)$ domains of quality of life. The program of DMT showed significant effects on minimizing perceived stress

Ho et al. ${ }^{30} \quad(p<0.050)$, in women with breast cancer during radiotherapy treatment, and also showed significant effects in pain severity and pain interference $(p<0.050)$.

Ho et al. ${ }^{33} \quad$ Better results in coping with cancer, mental well-being, attention, and appreciation for the self and body.

Not investigated.

The study group (S) demonstrate improvements in physical aspects of quality of life $(p<0.001)$.

Not investigated.

The breast cancer patients indicate improvement in total functioning and physical symptoms. 


\section{Discussion}

Different psychological benefits were presented in the studies of this review after dance intervention. The 11 selected studies have indicated positive improvements in quality of life $e^{25,30,33}$, decreased fear of disease recurrence, improvement in the attitude, feelings of freedom and joy for dance practice ${ }^{28}$ and also the perceived stress and pain $^{30}$, pointing out that the negative feelings involved in the context of these women, were suppressed, minimized and released through body movements ${ }^{27}$. The authors found an increase of $36.3 \%$ on satisfaction with lifestyle and reduction in $35 \%$ of depressive symptoms in patients after intervention ${ }^{24}$. Still, patients have reported a modified and healthy view in relation to body image and feelings of femininity, grace and sensuality ${ }^{23}$. The patients from Ho et al..$^{33}$ study declared a bridge back to a normal and better life and participate in shared positive experiences after dance intervention. Pisu et al. ${ }^{26}$ revealed improvement in dyadic trust scale after ballroom dance doing in couples. Which indicates that dance interventions can be beneficial to some psychological aspects of breast cancer women.

When related to physical benefits, the anatomical region of the upper limbs was presented as an investigative focus in the studies of Sandel et al. ${ }^{31}$, which indicates an improvement in range of motion (ROM) of the upper limbs through the practice of dance, and Kaltsatou et al. ${ }^{24}$, pointed an improves of $24.3 \%$ for hand grip strength on the right side, and also $26.1 \%$ for the left side, even showing a decreases in the arm circunference of the mastectomized side. The findings from Molinaro et al. ${ }^{23}$ corroborate the revealing of strength maintenance and ROM of upper limbs qualitatively pointed by women. Other physical variable investigated was functional capacity in the study of Pisu et al. ${ }^{26}$, and the results showed improvements after dance intervention. Still, the authors suggested that such parameters should be best investigated considering the importance of this variables regarding the kinetic and functional benefits and improvement in independence of daily life.

Serlin et al..$^{32}$ pointed out the need for more daily and qualitative investigations, in order to analyze possible improvements at every session, and not necessarily after the 12 suggested sessions, because some participants held chemotherapy or radiotherapy in the process which modifies their feelings towards dancing, and participation only in a few sessions. Also regarding to social aspects, Sandel et al. ${ }^{31}$ pointed out the reduction feeling of isolation, especially in women going through chemotherapy. Likewise, Ho et al. ${ }^{30}$ investigated dance during radiotherapy, and affirmed that they encouraged interactions, emotional expressions and communications between the participants of the intervention group, with the moments of sharing experiences after the daily intervention. Thus, it is understood that dance practice in breast cancer women promotes psychological well-being, even in its different stages of the treatment.

The studies regarding dance modality selected for interventions have not found hegemony. Mostly, specific methods of dance therapy, such as The Lebed Method used by Sandel et al. ${ }^{31}$, the Mindfull Movement Program by Crane-Okada et al. ${ }^{28}$, Dance Movement Therapy (DMT) by Blázquez et al. ${ }^{27}$, Ho et al. ${ }^{30}$ and Ho et al. ${ }^{33}$. Authentic Movement by Dibbel-Hope et al. ${ }^{29}$, and Kinaesthetic Imagining by Serlin et al. ${ }^{32}$. In contrast, Molinaro et al. ${ }^{23}$ feature one of the first reports about dance for breast cancer patients, using techniques known worldwide as classical ballet and jazz, Kaltsatou et al. ${ }^{24}$ with Greek traditional dance associated with the training of upper limbs, Frison et al..$^{25}$ with sacred dance and Pisu et al. ${ }^{26}$ with ballroom dance. Future studies are suggested to compare the effects of different forms of dance therapy, which would allow the most appropriate selection for 
each one of these women as the rhythm, movement type, dance style and personal peculiarities.

The variability in the intervention period (three weeks to 24 weeks), in weekly frequency (one to three sessions a week) and duration of the session (one to three hours), can be justified by the different methodological strategies of studies, availability of patients, within their limits and treatment, as well as related to the place of intervention. Concerning the issue, the American College of Sports Medicine (ACSM) recommends for patients with cancer, 150 minutes of moderate physical activity or 75 minutes of vigorous physical activity per week, as well as resistance training twice a week, in order to improve general physical health ${ }^{34}$. Dance classes with one hour length three times a week, or three hours length once a week, may be considered to be within the ACSM recommendations.

Regarding the basics structure of the interventions just two studies distinguish them ${ }^{24,31}$. Sandel et al. ${ }^{31}$ feature a warm-up and basic exercises for upper limbs (< 15 minutes), the main part with dance moves (<30 minutes), finishing with the relaxation/calm down ( $<10$ minutes). Kaltsatou et al. ${ }^{25}$ also used warm-up $(<10$ minutes), the main part with aerobic training and learning the dance steps of Greek sacred dance (<25 minutes), followed by the upper limb strength training and calm down ( $<25$ minutes). They differ only in the main part, since they are distinct methodologies, with similarities in warm-up and calm down. Such intervention structures can be replicated in clinical practice, however, it is recommended the development of protocol studies for better understanding.

The others nine studies did not presented deitaled structure for the dance intervention, they showed only the kind of approaches they used. Sandel et al. ${ }^{31}$, aimed to activate existential challenges and feelings experienced in the diagnosis and treatment, such as body image, sexuality, sensory control, the meaning of life, sadness and loss during all the dance classes. Blázquez et al. ${ }^{27}$, employed dynamic integration of body and mind through the use of motion experiences, for creative expression and by the integration of relaxation techniques and images.

As well as Dibbel-Hope et al. ${ }^{29}$, when the patients sat in a circle on the floor and each one began the class sharing their feelings about whatever was going on her life that week. After this part, the patients move around about 20 minutes while the leader witnessed, and for the rest of the session, is was used variations on this format: Moved in dyads with one mover and one witness; they moved in triads with one mover and two witnesses; half the group moved while half the group witnessed; one person moved while the group witnessed; and the entire group moved together at first, then gradually individuals moved out into the witness circle. After all that, the group share the experiences and talk about the class. In all the classes, the body of the individual moves on its own time and pace, under its own momentum, moving comfortably and without physical limitations ${ }^{29}$.

Crane Okada et al. ${ }^{28}$, used conscious walking and movements, verbal feedback, exploration of specific parts of the body, short, light and slow movements, movements with greater effort and intention, active movements with energy, and some work in pairs. And also, the leader used verbal feedbacks, often with music, and the patients were encouraged to move their bodies in ways that were fun, comfortable and creative. Ho et al. ${ }^{30}$ includes stretching, relaxation exercises, movement games and rhythmic body movement to exercise the upper extremities, improvisation and movement to explore positive emotions. Dance therapy methods are generally similar, with subjective approaches of perception of the body and movement fluency in relation to patient's feelings. The classes were all very specific for the 
group, with sharing experiences and moving across the rhythm of each women.

Guided by dance established methodologies, Kaltsatou et al. ${ }^{24}$, included aerobic training with traditional Greek dance practice, learning and practice of predetermined sequential movements. Similar behavior was adopted by Molinaro et al. ${ }^{23}$ to opted for classical ballet with the intention of working in isolation and symmetrically the upper limbs movements, still promoting a full body stretch, with improved posture and balance. Followed by the school of jazz, which emphasized range of motion exercises, with emphasis on the major and minor pectoral muscles, the shoulder joint, the neck and the trunk musculature in general. Frison et $a .^{25}$ with the sacred dance that occurs with a circle, all the women holding hands moving around the circle together. And Pisu et al. ${ }^{26}$ with ballroom dance for couples, wich mean, breast cancer survivor and their married partner, and it was used some specific type of ballroom dance: Foxtrot, Waltz, Cha-Cha, and East Coast Swing. All interventions, whether objective or subjective, showed benefits to the research public. It is understood that the choice between the various approaches is a woman decision on her own according to their familiarity and personal preferences to the dance styles.

Sandel et al. ${ }^{31}$, unveil questions about new studies that answer the question: at which moment of the treatment dance intervention will be more beneficial? The patients suggested that the intervention may happen soon after surgery. In this sense, Dibbel-Hope ${ }^{29}$ and Serlin et al. ${ }^{32}$ pointed out the importance of quali-quantitative approaches in order to explore different perceptions and not dissipate important information regarding subjective responses. Ho et al. ${ }^{33}$ investigated dance during radiotherapy and postradiotherapy, and concluded that the two groups can have benefitial from dance practice. Also, the radiotherapy group declare that dance help them to cope with the radiation treatment, either by speeding up their recovery from the current and previous treatments or by making the radiation period and adverse effects more bearable by giving them something to look forward. This found can indicate that dance intervention can be applied in any stage of breast cancer treatment.

Limitations of the studies are pointed as the small number of women investigated and the heterogeneity of the samples, making it impossible for conclusive results and the generalization to population. Another potential limitation was the time of intervention, since the learning of specific dance technique requires a longer time of lessons, for women's adherence to the activity, a relevant factor is the intimacy with the group, which could help to inhibit the timidity, and make movements free and automatic.

A difference between the design of the studies can be observed, which hinders or even prevents the comparison of studies on the theme. A need for studies with defined protocols, randomized, controlled and also, longitudinal methodologies is suggested, in order to find plausible evidence of reproducibility in clinical performance, and also investigating the maintenance of physical and psychological effects of dance in this population. It is also recommended studies that investigate the influence of dance on the physical and emotional complications in women who underwent different surgical techniques, or differents treatments, as chemotherapy, radiotherapy and hormoniotherapy.

A limitation related to this systematic review can be pointed as the search for the studies was too specific, it only considered the presence of keywords in title and abstract. In this way some potential articles could not be included in this systematic review. 
In looking at the studies entailing the present review, it was possible to state that dance, on different approaches, can promote psychological benefits in women with breast cancer. Such as, through the practice of classical ballet and jazz, with the encouragement of feelings of femininity, grace, and sexuality in relation to body image, dance therapy have been showing positive results in physical and mental well-being, the mood change and anxiety, in quality of life, on body image, Greek dance with decreases of depressive symptoms and improvement in life satisfaction, and also, ballroom dance in couples, that can enhance the confidende between the partners. Physical improvements were also identified, and aimed as pointed as result of dance practice, with the increase in range of motion of the upper limbs and functional capacity. Nevertheless, influence and the use of different evaluation tools on physical variables are still uncertain and need more attention.

Dance can represent a psychotherapeutic treatment and a form of physical activity, based on body awareness, expression and acceptance, in order to facilitate physical, emotional, cognitive, and spiritual integration ${ }^{29}$. Therapeutic groups of patients with the same disease and experiencing similar feelings may diminish the sense of solitude and incomprehension before the others ${ }^{31}$. As conclusion of this systematic review is possible to declare that differents types of dance can improve physical and psychological life aspects of women with breast cancer.

\section{Author's contribution}

Each author contributed individually and significantly to the development of this manuscript. L. Boing (0000-0003-4978-9703)* was attributed to the conception, design, data collection, analysis and interpretation of data, writing the manuscript, and approval of the submitted version. A. D. Rafael (0000-0003-2596-2356)* H. Braga (0000-0001-5068-7021)*, F. F. Sperandio (0000-0002-2435-0246)* and A. J. P. Moraes (0000-0001-7421-6662)* contributed to the design, analysis and interpretation of the data, writing of the manuscript and approval of the presented version. A. C. A. Guimarães (0000-0001-5167-2921)* was attributed to the conception, design, data collection, analysis and interpretation of data, writing the manuscript, and approval of the submitted version. *ORCID (Open Researcher and Contributor ID).

\section{Conflict of interest}

The authors declare that they have no conflict of interest.

\section{References}

1. World Health Organization. (Ed.). World Health Statistics. Genebra. 2014. Disponible: <Http://Www.Who.Int/En/>. Acess: 01 august. 2014.

2. Brasil. Instituto Nacional do Câncer. Ministério da Saúde. Tipos de Câncer. 2014. Avaliable at: <http://www2.inca.gov.br/wps/wcm/connect/tiposdecancer/site/home>. Acess: 01 august. 2014.

3. World Health Organization (Ed.). Global Health Estimates: Breast Cancer.. Avaliable at: <http://www.who.int/healthinfo/global_burden_disease/en/.>. Acess: 01 august 2014.

4. Conde DM, Pinto-Neto AM, Freitas Junior R, Aldrighi JM. Qualidade de vida de mulheres com câncer de mama. Rev Bras Ginicol Obstet. 2005;28(3):195-204.

5. Mcneely ML, Campbell K, Ospina M, Rowe BH, Dabbs K, Klassen TP, et al. Exercise interventions for upper-limb dysfunction due to breast cancer treatment. Cochrane Database Syst Rev. 2010; CD005211.

6. Adamsen L; Quist M, Moller T, Herrstedt J, Kronborg D, Baadsgaard MT, et al. Effect of a multimodal high intensity exercise intervention in cancer patients undergoing chemotherapy: randomized controlled trial. BMJ. 2009;339:b3410. 
7. Vidor C, Leroyer A, Christophe V, Seillier M, Foncel J, Maele JV, et al. Decrease social inequalities return-to-work: development and design of a randomized controlled trial among women with breast cancer. BMC Cancer. 2014;14(267):1-9.

8. Luz ND, Lima ACG. Physical therapy resource in post-mastectomy lymphedema: a review of the literature. Fisioter Mov. 2011;24(1):191-200.

9. Avci IA, Kumcagiz H, Altinel B, Caloglu A. Turkish Female Academician Self-Esteem and Health Beliefs for Breast Cancer Screening. Asian Pac J Cancer Prev. 2014;15(1):155-60.

10. Ha EH, Cho YK. The Mediating Effects of Self-Esteem and Optimism on the Relationship between Quality of Life and Depressive Symptoms of Breast Cancer Patients. Psychiatry Investig. 2014;22(4):437-45.

11. Courneya KS. Exercise interventions during cancer treatment: biopsychosocial outcomes. Exerc Sport Sci Rev. 2001;29(2):60-4.

12. Courneya KS. Exercise in cancer survivors: an overview of research. Med Sci Sports Exerc. 2003;35(11):1846-52.

13. Schmitz KH, Holtzman J, Courneya KS, Mâsse LC, Duval S, Kane R. Controlled physical activity trials in cancer survivors: a systematic review and meta-analysis. Cancer Epidemiol Biomarkers Prev. 2005;14(7):1588-95.

14. Sturm I, Baak J, Storek B, Traore A, Thuss-Patience P. Effect of dance on cancer-related fatigue and quality of life. Support Care Cancer. 2014;22:2241-49.

15. Prado MAS, Mamede MV, Almeida AM, Clapis MJ. Physical training practice in women submitted to breast cancer surgery: perception of barriers and benefits. Rev Latinoam Enfermagem. 2004;12(3):494-502.

16. Speck RM, Courneya KS, Mâsse LC, Duval S, Schmitz KH. An update of controlled physical activity trials in cancer survivors: a systematic review and meta-analysis. J Cancer Surviv. 2010;4(2):87-100.

17. Czepa, D, Ravenstein S, Stäuber F, Hilberg T. Development and evaluation of a dancebased exercise therapy for patients with haemophilia. Hamostaseologie. 2013;33:S25-31.

18. Vankova H, Holmerova I, Machacova K, Volicer L, Veleta P, Celko AM. The Effect of Dance on Depressive Symptoms in Nursing Home Residents. J Am Med Dir Assoc. 2014;15(8):582-87.

19. Mcgill A, Houston S, Lee R. Dance for Parkinson's: A new framework for research on its physical, mental, emotional, and social benefits. Complement Ther in Med. 2014;22(3):426-32.

20. Green S et al. 2011 Chapter 1: Introduction. In: Higgins JPT, Green S (editors), Cochrane Handbook for Systematic Reviews of Interventions. The Cochrane Collaboration Version 5.1.0. [updated March 2011] <www.cochrane-handbook.org>. Accessed 01 ago 2014.

21. Moher D, Liberati A, Tetzlaff J, Altman D. Preferred reporting items for systemat-ic reviews and meta-analyses: The PRISMA statement. Plos Med 2009;6(7): e1000097.

22. Downs SH, Black N. The feasibility of creating a checklist for the assessment of the methodological quality both of randomized and nonrandomized studies of health care interventions. J Epidemiol Community Health. 1998;52,377-84.

23. Molinaro J, Kleinfelf M, Lebed S. Physical Therapy and Dance in the Surgical Management of Breast Cancer: A clinical report. Phys Ther. 1986;66:967-69.

24. Kaltsatou A, Mameletzi D, Douka S. Physical and psychological benefits of a 24-week traditional dance program in breast cancer survivors. J Bodyw Mov Ther. 2011;15:162-67.

25. Frison FS, Shimo AKK, Gabriel M. Dança circular e qualidade de vida em mulheres mastectomizadas: um estudo piloto. Saúde Debate 2014;38(101):277-84.

26. Pisu M, Demark-Wahnefried W, Kenzik KM, Oster RA, Lin CP, Manne S, Alvarez R, Martin MY. A dance intervention for cancer survivors and their partners (RHYTHM). J Cancer Surviv. 2017;11(3)350-59.

27. Blázquez A, Nierga J, Javierre C. Terapia A Través De La Danza Y El Movimiento En Mujeres Con Cáncer De Mama: El Impacto Psicofísico Del Proceso Empático. Psicooncología. 2009, 8(1):31-43.

28. Crane-Okada R, Kiger H, Sugerman F, Uman GC, Shapiro SL, Wyman-McGinty W, Anderson NLR. Mindful Movement Program for Older Breast Cancer Survivors - A Pilot Study. Cancer Nurs. 2012;35(4):E1-E13.

29. Dibbell-Hope S. The Use Of Dance/Movement Therapy In Psychological Adaptation To Breast Cancer. Arts Psychother. 2000;27(1):51-68. 
30. Ho RTH, Fong TCT, Cheung IKM, Yip PSF, Luk M. Effects of a Short-Term Dance Movement Therapy Program on Symptoms and Stress in Patients With Breast Cancer Undergoing Radiotherapy: A Randomized, Controlled, Single-Blind Trial. J Pain Symptom Manage 2016;51(5):824-31.

31. Sandel SL, Judge JO, Landry N, Faria L, Ouellette R, Majczak M. Dance and Movement Program Improves Quality-of-Life Measures in Breast Cancer Survivors. Cancer Nurs. 2005;28(4):301-09.

32. Serlin I, Classen C, Frances B, Angell K. Symposium: Support Groups For Women With Breast Cancer: Traditional And Alternative Expressive Approaches. Arts Psychother. 2000;27(2):123-38.

33. Ho RTH, Lo PHY, Luk PMY. A Good Time to Dance? A Mixed-Methods Approach of the Effects of Dance Movement Therapy for Breast Cancer Patients During and After Radiotherapy. Cancer Nurs. 2016;39(1)32-41.

34. Schmitz KH, Courneya KS, Matthews C, Demark-Wahnefried W, Galvao DA, Pinto BM. American College of Sports Medicine roundtable on exercise guidelines for cancer survivors. Med Sci Sports Exerc. 2010;42(7):1409-26.

Corresponding

Author

Leonessa Boing

leonessaboing@gmail.com
Rua Desembargador Pedro Silva,

2034, bl 03 apto 04, Coqueiros. CEP:

88080700. Florianópolis, SC
Received $\quad 05 / 12 / 2016$

Revised 23/03/2017

26/04/2017

Approved 27/04/2017 